\title{
IVD.02 - Identification and experimental validation of immunodominant B- cell epitopes present on hantavirus genotypes associated to hemorrhagic fever and renal syndrome
}

Fernando de Paiva Conte ${ }^{1 *}$; Thiago Santos Chaves'; Thais da Silva Araujo'; Renata Carvalho de Oliveira ${ }^{1}$; Elba Regina Sampaio Lemos ${ }^{1}$; Patrícia Cristina da Costa Neves ${ }^{1}$; Rodrigo Nunes Rodrigues da Silva ${ }^{1}$.

1Fiocruz/Bio-Manguinhos.

Introduction: Hantavirus infection is a worldwide neglected zoonotic disease, affecting 200,000 people/year, mainly transmitted to humans through the inhalation of contaminated aerosols, saliva and/or urine of infected rodent animals. The clinical manifestations of the disease in humans mainly include hemorrhagic fever with renal syndrome (HFRS) and hantavirus cardiopulmonary syndrome (HCPS). These syndromes are correlated to hantavirus genotypes and they are intimately associated to rodent reservoirs that have a specific geographical distribution worldwide. HFRS is caused by Old World hantaviruses, like Seoul virus. However, despite the report frequent occurrence of HFRS in Eurasia and infrequent diagnosis of disease in Americas, Seoul virus was isolated in rodent samples and specific antibodies against this genotype were found in serum from patients with suspected leptospirosis in Brazil. On this context, the presence of Rattus norvegicus allied to the previous report of Seoul virus in Brazilian territory, remarks however the urgency of development of novel diagnostic tools in order to detect hantavirus genotypes associated with HFRS, to avoid the misdiagnosis of this syndrome as other bacterial or viral hemorrhagic fevers. Besides, hantavirus nucleocapsid protein (NP) is the major antigen that elicits early serological responses in infected humans and has been used as a biomarker to develop antibodies for epidemiological surveillance in regions where various hantavirus species cocirculate. However the identification of B-cell epitopes on NP remains unexplored.

Objective: To identify immunogenic B-cell linear epitopes in the nucleocapsid of hantavirus genotypes associated to HFRS.

Methodology: In silico analysis were performed using the Seoul nucleocapsid sequence (SNP) obtained from NCBI. Linear B-cell-epitopes on SNP and its immunogenicity were predicted by BepiPred-2.0 and Vaxijen algorithms, respectively. The predicted epitopes were aligned to nucleocapsid sequences of hantavirus genotypes associated to HFRS (Gou, Hantaan, Amur and Dobrava-Belgrade) and HCPS (Juquitiba, Andes, Jabora, Laguna Negra and Sin Nombre) to compare the degree of conservation among hantavirus-genotypes. The B-cell-epitopes only conserved among HFRS genotypes were synthetized as linear peptides and used to experimental validation, by the evaluation of serum reactivity from $\mathrm{BalB} / \mathrm{c}$ mice intraperitoneally immunized with purified recombinant SNP emulsified in complete Freund's adjuvant on day zero and boosted on days 14 and 28 in incomplete Freund's adjuvant. Mice IgG serum titration were assayed at days 21 and 35 using ELISA against therecombinant-SNP or B-cell predicted-epitopes.

Results: The in silico analysis resulted in 9 potential B-cell epitopes on SNP. Focusing on epitopes highly conserved just among HFRS genotypes, we found epitopes with moderate (HAN002) and low (HAN-005 and HAN-006) degrees of conservation in comparison with HCPS genotypes, which were selected to further experimental validation. Sera from mice immunized with recombinant-SNP showed significant IgG titers against HAN-002 and HAN-005 peptides.

Conclusion: The epitope HAN-005 is a potential candidate for development of better diagnostic tools for hantavirus genotypes associated to HFRS

Keywords: B-cell epitopes; hemorraghic fever; Seoul hantavirus 